\title{
Protocolo terapêutico para cirrose hepática canina - uso em três animais
}

\section{Therapeutic protocol for canine liver cirrhosis - use in three animals}

\section{Carlos Eduardo Fonseca-Alves ${ }^{[a]}$, Fabiana Elias $^{[\mathrm{b}]}$, Helvécio Leal Santos Junior ${ }^{[\mathrm{b}]}$}

\section{Resumo}

A cirrose é a alteração crônica e irreversível do fígado caracterizada por um grau de fibrose e regeneração que resulta na desorganização da arquitetura hepática. Para o diagnóstico, a biópsia hepática faz-se necessária, confirmando os achados clínico-laboratoriais além de auxiliar na avaliação prognóstica. Atendeu-se no serviço de clínica médica do Hospital veterinário três cães apresentando: ascite (3/3), hiporexia (2/3), icterícia (1/3), vômito (2/3) e dispneia (2/3). Após diagnóstico instituiu protocolo terapêutico com colchicina $0,03 \mathrm{mg} / \mathrm{kg}$ SID, Silimarina a $70 \mathrm{mg} / \mathrm{kg}$ BID, ácido ursodesoxicólico a $5 \mathrm{mg} /$ $\mathrm{kg}$ BID, Furosemida a $2 \mathrm{mg} / \mathrm{kg}$ BID, suplemente de vitamina E a $5 \mathrm{mg} / \mathrm{kg}$ SID, vitamina C a $20 \mathrm{mg} / \mathrm{kg}$ BID e dieta a base arroz, frango, batata e cenoura. 0 protocolo proposto foi eficiente ao causar remissão dos sinais clínicos, proporcionando melhor qualidade de vida aos animais. Mais estudos são necessários para comprovar a eficiência desses medicamentos no tratamento da cirrose canina.

Palavras-chave: Cão. Cirrose. Tratamento.

\begin{abstract}
Cirrhosis is a chronic and irreversible change of the liver characterized by a degree of fibrosis and regeneration that results in disruption of the hepatic architecture. Liver biopsy is necessary for the diagnosis, thus confirming the clinical and laboratorial findings besides helping in the prognostic assessment. Three dogs suffering from ascites (3/3), appetite loss (2/3), jaundice (1/3), vomiting (2/3) and dyspnea (2/3) were attended in the internal clinical ward of the Veterinarian Hospital. After the diagnosis a treatment protocol was established with use of colchicine $0.03 \mathrm{mg} / \mathrm{kg}$ SID, Silymarin $70 \mathrm{mg} / \mathrm{kg}$ BID, ursodeoxycholic acid at $5 \mathrm{mg} / \mathrm{kg}$ BID, furosemide, $2 \mathrm{mg} / \mathrm{kg}$ BID, vitamin E supplement at $5 \mathrm{mg} / \mathrm{kg}$ SID, Vitamin C at $20 \mathrm{mg} / \mathrm{kg}$ BID and a rice, chicken, potatoes and carrots based diet. The proposed protocol was effective in causing remission of clinical signs, providing better quality of life for those animals. More studies are needed to prove the efficiency of those medicines in the treatment of canine cirrhosis.
\end{abstract}

Keywords: Dog. Cirrhosis. Treatment.

\section{$\sqrt{10}$}

[a] Mestrando do programa de Pós Graduação em Medicina Veterinária, DCV, Universidade Estadual Paulista "Júlio de Mesquita Filho", Botucatu, São Paulo, Brasil, e-mail: carloseduardofa@hotmail.com

${ }^{[b]}$ Professora adjunta do curso de Medicina Veterinária, Universidade Federal da Fronteira Sul (UFFS), Realiza - PR, Brasil.

Recebido: 05/03/2010

Received: 03/05/2010

Aprovado: 14/10/2010

Approved: 10/14/2010 


\section{Introdução}

A cirrose é definida como uma alteração crônica e irreversível do fígado, caracterizada por um grau de fibrose e regeneração que resulta na desorganização da arquitetura hepática (1). Dentre os sinais clínicos associados incluem anorexia, depressão, perda de peso e icterícia (2). Para o diagnóstico, a biópsia hepática faz-se necessária, confirmando os achados clínico-laboratoriais além de auxiliar na avaliação prognóstica (3).

0 tratamento de afecções hepáticas crônicas consiste em terapia de suporte, incluindo uso de dietas modificadas e medicações específicas administradas para diminuir o processo fibrótico (2). 0 tratamento envolve agentes antioxidantes, citoprotetores, antinflamatórios e antifibróticos. Esses agentes aprimoraram os mecanismos de defesa natural para inibir a inflamação e fibrose, prevenir a apoptose, ou proteger contra a lesão oxidativas pela manutenção de um equilíbrio de redução adequado (1).

O prognóstico é desfavorável, pois não se pode eliminar a causa primária. 0 presente trabalho tem por objetivo descrever um protocolo terapêutico proposto para tratamento da cirrose em cães.

\section{Material e métodos}

Atenderam-se no serviço de clínica médica do Hospital Veterinário três cães apresentando: ascite $(3 / 3)$ hiporexia $(2 / 3)$, icterícia $(1 / 3)$, vômito $(2 / 3)$ e dispneia $(2 / 3)$. Por causa do desconforto apresentado pelos animais, inicialmente optou-se pela drenagem do líquido abdominal (3/3). Realizou-se radiografia abdominal, ultrassonografia, hemograma, bioquímica hepática, renal e avaliação do líquido ascítico nos três animais.

Após diagnóstico, foi instituído protocolo terapêutico com colchicina $0,03 \mathrm{mg} / \mathrm{kg}$ SID, Silimarina a $70 \mathrm{mg} / \mathrm{kg}$ BID, ácido ursodesoxicólico a $5 \mathrm{mg} / \mathrm{kg}$ BID, Furosemida a $2 \mathrm{mg} / \mathrm{kg}$ BID, suplemente de vitamina E a $5 \mathrm{mg} / \mathrm{kg}$ SID, vitamina C a $20 \mathrm{mg} / \mathrm{kg}$ BID e dieta a base de arroz, frango, batata e cenoura.

\section{Resultados e discussão}

A consequência mais grave das lesões hepáticas é a insuficiência hepática, que pode ser causada por destruição hepática súbita. Com maior frequência, a insuficiência hepática é o desfecho de uma lesão progressiva do fígado, seja por destruição insidiosa de hepatócitos ou por ataques repetidos de lesão ao parênquima hepático (1). Os animais do presente relato tinham 7, 9 e 10 anos. Em um estudo retrospectivo, $50 \%$ dos animais foram classificados como idosos, $48,6 \%$ como adultos e 1,4\% como filhotes reiterando que animais adultos e idosos têm maior possibilidade de desenvolver cirrose (4).

Nos animais aqui apresentados, não se encontrou possível etiologia. É relatado que, embora várias doenças hepáticas crônicas possam culminar em cirrose, é difícil estabelecer a etiologia considerando critérios histológicos e em muitos casos os proprietários não revelam informações que possam auxiliar (5).

Os principais sinais clínicos apresentados foram ascite $(3 / 3)$ hiporexia $(2 / 3)$, icterícia $(1 / 3)$, vômito $(2 / 3)$ e dispneia (2/3). A insuficiência hepática ocorre quando há comprometimento de pelo menos $70 \%$ a $80 \%$ da massa hepática funcional, excedendo sua capacidade de reserva funcional, resultando em sinais clínicos de insuficiência hepática (4). A ascite é um sinal clínico comum e resulta de hipertensão portal, ativação neuro-hormonal ou hipoalbulminemia. Os mesmos autores, avaliando parâmetros histológicos e variações clínicas, associaram a ascite como um fator prognóstico desfavorável, com animais apresentando poucos dias de vida.

A avaliação radiográfica não apresentou grande auxílio diagnóstico em virtude da grande quantidade de líquido abdominal e impossibilidade de visualizar estruturas abdominais. A avaliação ultrassonográfica em todos os cães evidenciou presença de líquido abdominal, micro-hepatia, fígado com contornos irregulares e hiperecogênico. Os métodos de diagnóstico não invasivos são necessários, pois, em muitos casos, os animais não podem ser submetidos a procedimentos anestésicos para realização de biópsia (5), sendo a ultrassonografia como método de diagnóstico e acompanhamento não invasivo.

0 hemograma revelou anemia (2/3) (normocítica normocrômica) e na avaliação da fosfatase alcalina e alanina-aminotransferase, em dois animais apresentava-se dentro dos valores de referência e em um animal estava acima do valor de referência (150 e 220 respectivamente). A ureia e creatinina estavam dentro dos valores de normalidade em todos os animais. Em estudo retrospectivo encontrou-se anemia em $84,6 \%$ dos cães, $100 \%$ apresentaram aumento de ALT e em $75 \%$ FA estava aumentada (4). 
Dentre as drogas utilizadas no protocolo terapêutico, optou-se pela utilização da colchicina, um alcaloide que possui ação anti-inflamatória e antifibrótica (6). Em medicina humana, o colchicina faz parte de protocolos terapêuticos para várias afecções, inclusive cirrose hepática. Alguns autores comprovaram que animais com cirrose aumentam a lipoperoxidaçäo graças ao aumento de estresse oxidativo, sendo este, passível de redução após uso da colchicina (7).

A silimarina é um fitoterápico que impede o dano dos radicais livres atuando como antioxidante. A silimarina não só impede a depleção de glutationa (GSH) induzida porxinas hepáticas, mas demonstrou-se que aumenta GSH basal do fígado, sendo extremamente útil quando a exposição a substâncias tóxicas é alta, graças ao papel vital da glutationa nas reações de desintoxicação (8).

0 ácido ursodesoxicólico, em medicina humana, tem sido usado rotineiramente no tratamento de doenças hepáticas crônicas tais como cirrose biliar primária, colangite esclerosante e hepatite autoimune. Acredita-se que essa droga tenha um papel citoprotetor a partir de sua ação como estabilizadora de membranas celulares (9). Foi possível demonstrar melhora significativa dos parâmetros enzimáticos séricos de humanos, inclusive com diminuição da inflamação e retardo no avanço da fibrose, após os 12 meses de tratamento ácido ursodesoxicólico (10). Em experimento realizado anteriormente utilizou-se protocolos com colchicina, silimarina e ácido ursodesoxicólico conseguindo reduzir significativamente a peroxidação lipídica em ratos cirróticos, no entanto, constatou que a colchicina não teve ação antifibrótica (11).

Dieta especial para cães com cirrose é recomendada para fornecer os nutrientes e calorias necessárias para sustentar o reparo do tecido hepático e minimizando metabolismo proteico que induz a encefalopatia hepática. Foi prescrito ração comercial hepática, no entanto os animais mostraram-se relutantes em ingerir a ração (4). Optou-se então por utilizar dieta caseira com alto teor de carboidratos.

Dois pacientes obtiveram a média de sobrevida de 4,5 meses, sendo realizada necropsia e confirmando o diagnóstico (Figura 1). 0 terceiro paciente está a quatro meses em tratamento, apresenta-se estável sem sinais clínicos de doença hepática. As doenças crônicas que afetam cães permanecem com etiologia não estabelecida, sem tratamento específico. Quando há sinais avançados de insuficiência hepática, o prognóstico é desfavorável com animais apresentando poucas semanas de vida (5).
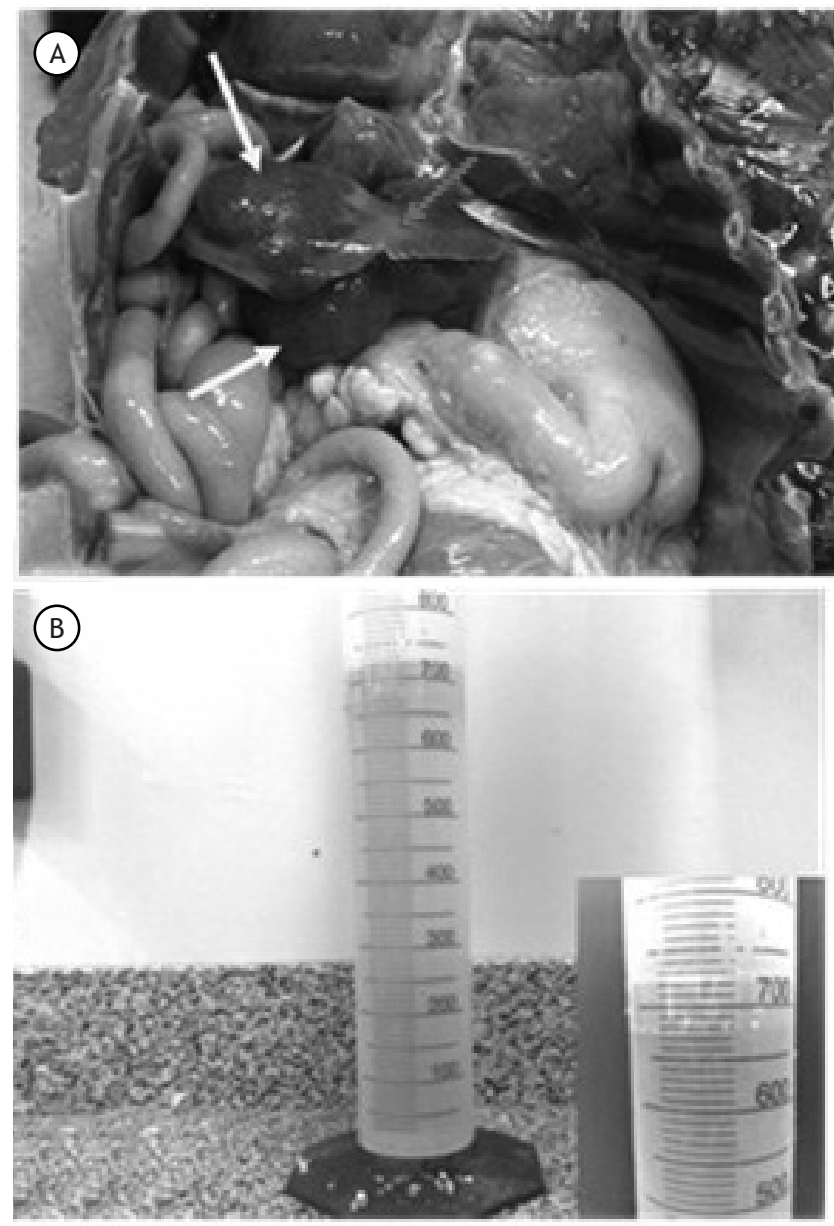

Figura 1 - A) Fígado diminuído de tamanho, de coloração amarelado amarronzado, irregular, com formações nodulares (seta branca) delimitadas por áreas esbranquiçadas de tecido conjuntivo fibroso (seta vermelha). B) Aproximadamente $720 \mathrm{~mL}$ de líquido ascítico amarelado proveniente do mesmo animal

Fonte: Setor de patologia animal - Faculdade UPIS.

\section{Conclusões}

A cirrose hepática é uma doença progressiva, com prognóstico desfavorável, e com poucos dados relacionando terapêutica e sobrevida em pacientes caninos. A colchicina em associação com a silimarina e o ácido ursodesoxicólico é frequentemente estudada em medicina humana, mas a sua eficiência na Medicina Veterinária é pouco relatada. $\mathrm{O}$ protocolo utilizado no estudo foi eficiente ao causar remissão dos sinais clínicos, proporcionando melhor qualidade de vida aos animais. Mais estudos são necessários para comprovar a eficiência de tais medicamentos no tratamento da cirrose canina. 


\section{Referências}

1. Webster CRL, Cooper J. Therapeutic use of cytoprotective agents in canine and feline hepatobiliary disease. Vet Clin North Am Small Anim Pract. 2009;39(3):631-52.

2. Reis KAA, Roquete MLV, Penna FJ. Esteatohepatite não alcoólica na infância e adolescência: revisão. Pediatria. 2001;23(4):329-39.

3. Horiguchi K, Hirano T, Ueki T, Hirakawa K, Fujimoto J. Treating liver cirrhosis in dogs with hepatocyte growth factor gene therapy via the hepatic artery. J Hepatobiliary Pancreat Surg. 2009;16(2):171-7.

4. Favier RP. Idiopathic hepatitis and cirrhosis in dogs. Vet Clin North Am Small Anim Pract. 2009;39(3):481-8.

5. Crawford JM. Liver and biliary tract. In: Kuman V, Abbas AD, Fausto N. Robbins and Cotran Pathologic Basis of disease. 7th ed. Philadelphia: Saunders; 2004. p. 877-937.

6. Lessa AS, Elia CCS, Rezende GFM, Resende CMC. Ultrassonografia na avaliação de um modelo experimental de esteatose e cirrose em ratos Wistar. Radiol Bras. 2007;41(2):98.
7. Rambaldi A, Gluud C. Colchicine for alcoholic and non-alcoholic liver fibrosis or cirrhosis. Liv Intern. 2001;21(2):129-36.

8. Favari L, Pérez-Alvárez V. Comparative effects of colchicine and silymarin on CCI4-chronic liver damage in rats. Arch Med Res. 1997;28(1):11-7.

9. Reis KAA, Roquete MLV, Penna FJ. Esteatohepatite nãoalcoólica na infância e adolescência: revisão. Pediatria. 2001;23(4):329-39.

10. Laurin J, Lindor KD, Crippin JS, Gossard A, Gores GJ, Ludwig J, et al. Ursodeoxycholic acid or clofibrate in treatment of non-alchol-induced steatohepatitis: a pilot study. Hepatology. 1996;23(6):1464-7.

11. Cremonese RV, Pereira-Filho AA, Magalhães R, Mattos AADE, Marroni C, Zettler CG, et al. Experimental cirrhosis induced by carbon tetrachloride inhalation: technical modifications and lipoperoxidation effects. Arch Gastroenter. 2001;38(1):40-7. 